\title{
Mapping of Krau Wildlife Reserve (KWR) protected area using Landsat 8 and supervised classification algorithms
}

\begin{abstract}
Human-dominated ecosystems speed up the loss of habitats, populations, and species. Thus, monitoring and managing the Earth's heritage of biodiversity is a challenge in natural resource management. Mapping protected areas (PAs) is essential in understanding the disturbance that can affect biodiversity and conservation management. Land use-land cover (LULC) maps can be used as a decision making tool by policy makers to ensure sustainable development and understanding of the effect of human activities within and around PAs. However, in Malaysia, the limited updated maps of PAs make the effective management of PAs problematic. Therefore, this study aimed to produce an updated Land LULC map for the PA Krau Wildife Reserve (KWR) and its surroundings using remote sensing and related geospatial technologies. Three supervised classification algorithms were used and compared. Multidated images from Landsat 8 were utilized, and spectral angle mapper (SAM), support vector machine (SVM), and artificial neural network (ANN) classifiers were applied and evaluated. The approaches of pan-sharpening and cloud patching were used to enhance the accuracy of LULC classification. The images were classified into five classes: dense forest, less dense forest or agriculture, builtup area, bare soil, and water. The overall accuracies of SAM, ANN, and SVM for the $15 \mathrm{~m}$ spatial resolution images were $81.96 \%, 98.22 \%$ and $97.40 \%$, respectively. The ANN map produced the highest overall accuracy and was consequently utilized to extract additional information related to disturbance and encroachment within and around the PA. Findings indicated that socioeconomic activities played a major role in altering the environment of KWR.
\end{abstract}

Keyword: LULC; Classification algorithms; Protected Area; Krau Wildlife Reserve; Geospatial technologies 